\title{
Bright Quantum Dot Single-Photon Source at 1.55 m Heterogeneously Integrated on Si
}

Holewa, Pawe; Sakanas, Aurimas; Gür, Ugur Meriç; Mrowiski, Pawe; Gregersen, Niels; Syperek, Marcin ; Semenova, Elizaveta

Published in:

Conference on Lasers and Electro-Optics

Link to article, DOI:

10.1364/CLEO_SI.2021.SM1Q.2

Publication date:

2021

Document Version

Peer reviewed version

Link back to DTU Orbit

Citation (APA):

Holewa, P., Sakanas, A., Gür, U. M., Mrowiski, P., Gregersen, N., Syperek, M., \& Semenova, E. (2021). Bright Quantum Dot Single-Photon Source at $1.55 \mathrm{~m}$ Heterogeneously Integrated on Si. In Conference on Lasers and Electro-Optics IEEE. https://doi.org/10.1364/CLEO_SI.2021.SM1Q.2

\section{General rights}

Copyright and moral rights for the publications made accessible in the public portal are retained by the authors and/or other copyright owners and it is a condition of accessing publications that users recognise and abide by the legal requirements associated with these rights.

- Users may download and print one copy of any publication from the public portal for the purpose of private study or research.

- You may not further distribute the material or use it for any profit-making activity or commercial gain

- You may freely distribute the URL identifying the publication in the public portal 


\title{
Bright Quantum Dot Single-Photon Source at $1.55 \mu \mathrm{m}$ Heterogeneously Integrated on $\mathrm{Si}$
}

\author{
Pawel Holewa $^{1,2}$, Aurimas Sakanas ${ }^{1}$, Ugur Meriç Gür ${ }^{3}$, Pawel Mrowiński ${ }^{2}$, Niels Gregersen ${ }^{1}$, Marcin \\ Syperek $^{2}$, Elizaveta Semenova ${ }^{1,4}$ \\ 1 DTU Fotonik, Technical University of Denmark, DK-2800 Kgs. Lyngby, Denmark \\ 2 Laboratory for Optical Spectroscopy of Nanostructures, Faculty of Fundamental Problems of Technology, \\ Wroctaw University of Science and Technology, Wyb. Wyspiańskiego 27, 50-370 Wroctaw, Poland; \\ 3 DTU Electrical Engineering, Technical University of Denmark, DK-2800 Kgs. Lyngby, Denmark \\ 4 NanoPhoton-Center for Nanophotonics, Technical University of Denmark, DK-2800 Kongens Lyngby, Denmark \\ pawel.holewa@pwr.edu.pl
}

\begin{abstract}
We demonstrate high single-photon purity from an InAs/InP QD sources on Si operating at $1.55 \mu \mathrm{m}$. The single-photon extraction efficiency reaches up to $10 \%$ using a simple mesa structure.
\end{abstract}

\section{Introduction}

Semiconductor quantum dots (QD) are well-controllable and highly-flexible solid-state sources of single-photons. The information encoded in a quantum state of a photon can be utilized in the schemes of quantum communication or quantum information processing [1]. In this respect, quantum dots emitting at $1.55 \mu \mathrm{m}$ are highly desirable for future single-photon generators due to their compatibility with the existing silica-fibre-based telecom networks, allowing for optical signal transfer for long distances. Widely studied epitaxially-grown InAs/GaAs QDs have proved excellent optical properties [1]. Although these QDs are recognizable as near-perfect single-photon emitters for quantum information processing purposes, it is challenging to achieve the emission at $1.55 \mu \mathrm{m}$ in this material system [2]. In contrast, the telecom-application-relevant spectral range at $1.55 \mu \mathrm{m}$ can be straightforwardly obtained with InAs QDs embedded in the InP matrix. These dots have recently shown a record value for a single-photon generation purity from a self-assembled semiconductor quantum dot [3]. Despite such achievements, as-grown InAs/GaAs and InAs/InP QDs have typical low photon extraction efficiency, not exceeding 1\%. This inherent property of as-grown nanostructures is related to the total internal reflection at the air-semiconductor interface prohibiting detection of large photon fluxes outside the material, which is necessary for the application purposes. For the InAs/GaAs QDs, the problem is addressed by the shaping of a photonic environment of a QD, placing it in nanopillar microcavities [4], using microlenses [5], or bull's eye cavities [6], all enabling high photon extraction levels. However, for the InAs/InP QDs, the same approach remains an issue. Up to date, the best photon extraction efficiency for InAs/InP QDs emitting at $1.55 \mu \mathrm{m}$ is on the level of $10 \%$, obtained at the cost of tremendous technological effort (a complicated series of etching steps) to have a QD in an optical horn structure [7].

In this communication, we present a more versatile and robust solution for achieving equally high $(\sim 10 \%)$ photon extraction efficiency with $\mathrm{InAs} / \mathrm{InP}$ QDs at $1.55 \mu \mathrm{m}$ with room for further improvement. Moreover, we show heterogeneous integration of the InAs/InP QD with the Si wafer. Our approach is potentially interesting for the production of high brightness, high purity single-photon sources for photonic-integrated utilizing a well-developed and cost-effective silicon platform.

\section{Results}

\section{1. Fabrication of the structure}

The structure was grown on a (001) InP substrate by metal-organic vapor-phase epitaxy. A layer of Stranski-Krastanov InAs QDs with low surface density was sandwiched in the middle of an InP layer grown on an InGaAs sacrificial layer. After a deposition of 100-nm-thick $\mathrm{SiO}_{2}$ followed by thin aluminum layer. The structure was bonded using benzocyclobutene (BCB) with the Si substrate. Subsequently, the InP substrate and sacrificial layer were removed. A pattern of $0.25-5-\mu \mathrm{m}$-sized mesas was fabricated by the electron-beam lithography, allowing studies of single emission lines from QDs. The scheme of the structure is shown in Fig. 1(a).

\section{2. Single-photon purity and extraction efficiency}

We study the optical properties of individual InAs/InP QDs integrated into the Si platform. The Hanbury Brown and Twiss interferometry performed on the onset of selected QD emitters in the bounded structure, within the spectral range of $\sim 1.5-1.6 \mu \mathrm{m}$, shows a good single-photon emission purity reflected in the low value of a second-order 
correlation function $\mathrm{g}^{(2)}(\tau=0)$. We find that the purity is high for all the investigated emitters and exceeds $90 \%$. The exemplary single-photon correlation trace measured under pulse excitation condition is shown in Fig. 1(b). The value of $\mathrm{g}^{(2)}(\tau) \approx 0$ is also maintained at the optical excitation power corresponding to the saturation of the charged exciton line. We investigate emission purity at elevated temperatures important for the high-temperature operation of a potential device. At $\mathrm{T}=80 \mathrm{~K}$ (above the liquid-nitrogen boiling point), the value of $\mathrm{g}^{(2)}(\tau)=0.25$ is obtained, which is a record value for the InAs/InP QD system [2].

We evaluate the extraction efficiency from the fabricated masa structures. In the absolute approach, we estimate the setup transmission efficiency and from the single-photon detector count rates we determined the photon extraction efficiency values up to $\sim 10 \%$ (Fig. 1c).

Based on the calculations of the extraction efficiency, we find an agreement with the measured values. In particular, we find that the effect of the photonic environment engineering by mesas is of minor importance compared to the metallic mirror effect.

(a)

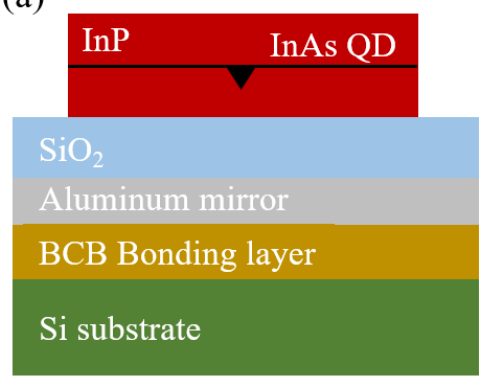

(b)

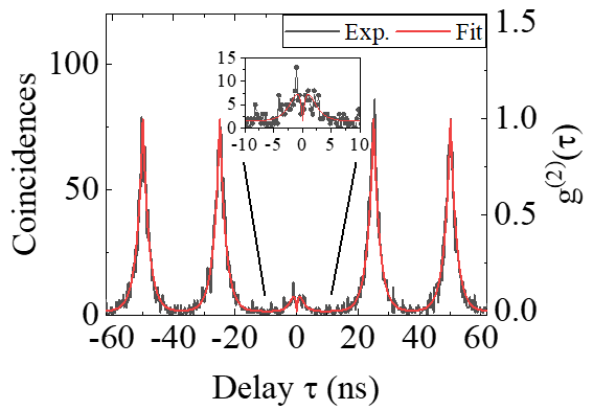

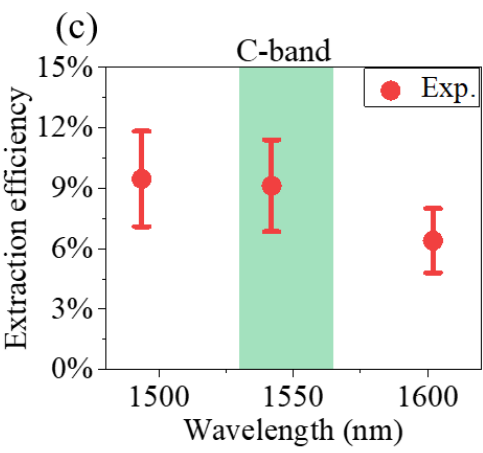

Figure 1 (a) Fabricated structure (InAs/InP QDs integrated with the Si wafer), (b) Autocorrelation of the exemplary single line emitting at $\mathbf{1 . 5 4} \boldsymbol{\mu \mathrm { m }}$, (c) Photon extraction efficiency for QDs in mesas.

\section{Summary}

In summary, we demonstrate high purity single-photon emission at $1.55 \mu \mathrm{m}$ from InAs/InP QD structure on $\mathrm{Si}$ platform. With our integration approach, the high extraction efficiency of $10 \%$ was achieved from a simple not optimized mesa structure. Further advancing of a cavity design will enhance the photon extraction efficiency close to unity.

\section{References}

[1] P. Senellart, G. Solomon, A. White, High-performance semiconductor quantum-dot single-photon sources. Nat. Nanotechnol., 12, 11 (2017).

[2] Y. Arakawa, M. Holmes, Progress in quantum-dot single photon sources for quantum information technologies: A broad spectrum overview. Appl. Phys. Rev., 7, 2 (2020).

[3] T. Miyazawa T, K. Takemoto, Y. Nambu, S. Miki, T. Yamashita, et al., Single-photon emission at $1.5 \mu \mathrm{m}$ from an InAs/InP quantum dot with highly suppressed multi-photon emission probabilities. Appl. Phys. Lett., 109, 13 (2016).

[4] H. Wang, Y. He, T. Chung, H. Hu, Y. Yu, et al., Towards optimal single-photon sources from polarized microcavities. Nat. Photonics, 13, 11 (2019).

[5] M. Gschrey, A. Thoma, P. Schnauber, M. Seifried, R. Schmidt, et al., Highly indistinguishable photons from deterministic quantum-dot microlenses utilizing three-dimensional in situ electron-beam lithography. Nat. Commun., 6, 7662. (2015)

[6] M. Davanço, M. Rakher, D. Schuh, A. Badolato, K. Srinivasan, A circular dielectric grating for vertical extraction of single quantum dot emission. Appl. Phys. Lett., 99, 4 (2011).

[7] K. Takemoto, M. Takatsu, S. Hirose, N. Yokoyama, Y. Sakuma, An optical horn structure for single-photon source using quantum dots at telecommunication wavelength. J. Appl. Phys., 101, 8 (2007). 